\title{
The Development of TLI (Thoracolumbar Lordotic Intervention) as an Effective Bracing Concept for the Postural Spinal Problems - A Review
}

\section{Piet Van Loon* and Ruud Van Erve}

Orthopedic Surgeons, Care to Move, Clinic for Orthopedic Assessment and Treatment, Deventer, The Netherlands

\begin{abstract}
Introduction: Gaps in knowledge in biomechanics on how the human spine develops itself to the adult posture still exist. It is clear that the huge increase in spinal problems do lack a sound etiologic base, on which effective preventive, conservative and surgical measurements should be based. An innovative technique to correct scoliosis and kyphosis in a non-surgical way is found in the TLI (thoracolumbar lordotic intervention) brace that is based on reversing of the external etiologic factors.
\end{abstract}

Method: A search in older or neglected orthopedic and biomechanical literature around spinal deformities was done to support the clearance of 'Idiopathy' in spinal deformities. Own research is described on the effectiveness of treatment by braces

Results: Older literature on postural development was gathered. Environmental factors like sitting were once investigated as causes for deformities and could be revisited. The vulnerability of young tissues and early deformability of especial the thoracolumbar spine (in school and child's labor) was known and well described. The work of Milan Roth on the essences of growth in the neuro-osseous growth relations showed itself a missing link in ongoing discussions on etiology. We related own observations on the detectable signs of raised tension in system during growth to the tension Roth described on cell biologic level. In the TLI brace technique, based on restoration of the crucial thoracolumbar lordosis in order to get healthy posture proof of the reproducibility of older knowledge could be given. The working mechanism of thoracolumbar lordotic intervention (TLI) was published and a pilot study with 91 children wearing this brace revealed good results and even true correction of scoliotic curves was reached. The events in the sagittal plane during (early) growth can be seen as decisive for the postures later in adulthood.

Keywords: Spine; Deformity; Scolisis; Kyphosis; Brace; Posture; Thoracolumbar spine

\section{Introduction}

TLI bracing is the result of a research and development effort, first in a local and personal setting, over about ten years. It was triggered by the discomfort in a general spinal surgery practice resulting from two issues:

The extensive practice in the last six decades on bracing for scoliosis did not lead to wide consensus in approach and method [1,2]. And the results showed poor in terms of correction. There was a consensus that bracing can stop the deformity progressing if started early enough, but not on evidence of relevant corrective growth. This leaves many patients with the ultimate consequence to be indicated for surgery: implanting an internal metal support structure that offers correction in radiologic studies, but brings fixation and thus limitation to the spinal functions. This solution has also some unsatisfactory elements, because in later life it can bring these patients much discomfort, not to speak about all perioperative risks [3]. For a physician, performing extensive surgery in otherwise healthy children is never an "easy job". It asks proper dealing with what is called in Psychology the cognitive dissonance: you offer a solution, but you have deep inside no or only little evidential support that it is the right one. At the other side the high quality of care and skills delivered will give mutual satisfaction on the results. But also in surgery in spinal deformities there is little consensus on approach and methods. In 25 years of personal experience in surgery for deformities the challenge to keep pace with methodical and industrial developments and innovations gave rise to the question where all this will end.

After centuries of progress in Medicine on causes of ailments, in the last century many orthopedic diagnoses got the adjective idiopathic attached to them. The lack of an understandable pathogenesis and remedies based on that has led to a dispersed conceptual thinking on possible causes, so a lot of undirected "trial and error" research and practice, the latter in both surgical as conservative forms, can be seen.

An extensive search in pre-Pub Med skeletal deformity related literature was done. Goal was to find if some helpful causative concepts were missed in time, or missed because of the limitations of the diagnostic techniques available in former days. And if so, can we formulate and apply consequences in treatment strategy.

\section{Characteristics of Scientific Progress in Earlier Research Periods}

In research in the pre WW I era, before the X-ray was used, very extensive scientific work can be found in German textbooks on the relation between posture and locomotion. Also was written down, that the correct position and capacity of musculature and its formative power (Form follows function) is dependent of a good balanced posture from the start. By the lack of $\mathrm{X}$-rays this was based on in vivo experiments, clinical observations (also epidemiological in schools) and by anatomical studies. Basic biologic knowledge and knowledge on evolutionary medicine, that evolved quickly in the nineteenth century was incorporated in orthopedic textbooks. These contain a lot of undisputed wisdom in basic orthopedics on normal morphogenesis

*Corresponding author: Piet Van Loon, Orthopedic Surgeons, Care to Move Clinic for Orthopedic Assessment and Treatment, Deventer, The Netherlands, Tel: 0031570760450; E-mail: pvanloon@caretomove.nl

Received January 09, 2015; Accepted April 24, 2015; Published April 26, 2015

Citation: Loon PV, Erve RV (2015) The Development of TLI (Thoracolumbar Lordotic Intervention) as an Effective Bracing Concept for the Postural Spinal Problems - A Review. J Spine 4: 226.doi:10.4172/21657939.1000226

Copyright: ( 2015 Loon PV, et al. This is an open-access article distributed under the terms of the Creative Commons Attribution License, which permits unrestricted use, distribution, and reproduction in any medium, provided the original author and source are credited. 
and pathology [4]. In 1912 Murk Jansen published his book (in Dutch) on the already accepted functional causes of scoliosis [5]. He brought a complete overview of knowledge on any aspect till then. It was the first and till our time the most impressive disclosure of the source of rotational forces in the human body that are needed to get a scoliosis and the relation between ventilation and spinal rotation in humans. He undertook different studies, the cadaveric with Robert Jones of Liverpool, that the asymmetric forces produced in ventilation by the asymmetric forces of the crurae diafragmaticae causes torque lumbar left sided and subsequently counter torque right sided at the thoracic spine. The way of description of the early "Sitzbuckel" or early kyphosis in siblings and toddlers is unique and with the clinical explanation that this ends in contractures at the thoracolumbar joint, could give a lasting explanation on the cause of scoliosis, if not the start of wars stopped this trail. But there no reference can be found in this era of growing knowledge on postural development on the active or passive role of the CNS in morphogenesis. There was strong awareness from Descartes on, of the important place of the brain and nerves in the complete function of the bodily system, but there was no insight in its intrinsic function or development in the phase of growth. The relationships between the CNS and the autonomic nervous system were known and also was depicted, that most of our balancing system and movements is based on reflexes (propriocepsis), independent of our cognitive brain But at the time Ramon y Cajal got the Nobel prize for the discovery of the synapse, unhappily the divergation between specialism's dealing with the two formative parts of the neuro-locomotor system was a fact. So the science concerning the musculoskeletal system lost contact with that concerning the neuromuscular system for more than a century.

The era of 1915 to 1945 shows no visible renewal or progress in methods or conceptual vision on spinal deformities by the tragic worldwide turmoil's that drove attention from doctors away from the normal growing child towards traumatology.

After WW II all research on scoliosis is pathomorphological and epidemiological in nature. The focus is on description of the curvature; $\mathrm{X}$-ray measuring is the method of choice in diagnostics, and the conservative approach with braces in which external forces directed at apical level to "push" back the curves are treatment. And later on surgical conservation gained popularity, especially by Harrington's invention of correction by intra-operatively expandable metal rods. An explosion in variety of implants followed [6]. But no imminent concept of the working mechanism of the still existent black box is formulated or tested: an explanation concerning how growth is organized and regulated (forces, masses, volumes) by the oldest organ of animal life: the central nervous system in vertebrates. Growth is mainly seen as a neurohormonal regulated increase in volume by mitosis, so that in the search for further clues in etiology of scoliosis the biochemical substances and genetics are in focus. But neuromuscular theories to explain the intriguing way our muscles give balance gain popularity [7]. Clinical assessment is on its way back: Cobb warned extensively at the time he popularized his radiologic curve measurements not to forget the clinical aspects of scoliosis [8].

\section{A Conceptual Breakthrough: The Work of Milan Roth (1923-2006)}

In the years 2003-2005 we rediscovered the complete work of Milan Roth. His work is totally overseen by the mainstream of western orthopedic and neuroscience. His starting point was the observations he did in scoliosis with air as contrast in myelographies (pneumomyelography). He worked behind the Iron Curtain and published mainly in Czech and German language in East European
Journals. Porter deserves the credit of first pointing to the value of this work for orthopedics [9]. We extensively examined the total work on his growth concepts. An overview of it on causes of spinal deformations was published in 2008 [10]. In his concept of the "Nervous Skeleton" and its way of growth Milan Roth developed between 1960 and 1985 his concepts on neurovertebral and neuro-osseous growth relations and the tension driven incongruence of growth (Figure 1) [11]. Roth provided biological experiments on how growth appears to support the older clinical observations in the nineteenth century. In animal experiments, mechanical modeling and radiological studies with air as contrast in scoliosis he stressed the role the growth by stretching of "the nervous skeleton" has in relation to the (ab-) normal formation of the spine/ skeleton by its mitotic based volume growth. The growth by stretching of the nervous system is a known high energy and oxygen demanding process. So is lack of oxygen is an initiator of deformities in Cerebral Palsy, because it destroys the stretch ability of axons. He was capable to induce scoliosis and other deformities in animals in teratogenic studies by intra-uterine injections of real inhibitors of neural growth and function. So a "short cord" can indeed cause scoliosis [12-15]. Although in kyphotic deformities asymmetry in the coronal plane is very common, but the same type of discongruency in growth relations is seen, there is little effort done to understand pathological postures as seen in Scheuermann "disease". Out of own unpublished material the same diminished caliber and tethered configuration of cord and roots, as well as concomitant Arnold Chiari malformations can be made visible on MRI in kyphotic children too. The stem-cord-roots complex is tethered, not by pathologic fibrous connections, but by intrinsic stiffening. Recent studies with MRI in idiopathic scoliosis supports this [16]. Neurosurgical articles on traction problems of the cord as in myelomeningocele do not refer to this intrinsic shortening depicted by Roth, but mainly on external tethering [17-19].

Roth deducted in a Socratic manner the way Wolff's Law and the Volkmann Hueter principle will work on a cellular level. Growth as an ongoing struggle to balance body masses against gravity has to create flat (Platyspondylie) or wedged vertebral bodies in hindered stretch growth of the cord leading to kyphotic and scoliotic deformities. Andry, van Gesscher and their followers were clear on the underlying relationship between sedentary lifestyle and the problems proper postural growth has to face (Appendix 1). Only now we can attach the European knowledge on how abnormal loading conditions in children on the elastic spinal column (children's labor, watchmakers, tailors and the sitting conditions in children) leading to the well-known deformities to his knowledge of the nervous growth: the optimal extending forces on the CNS are hindered by the cultural or lifestyle depending factors
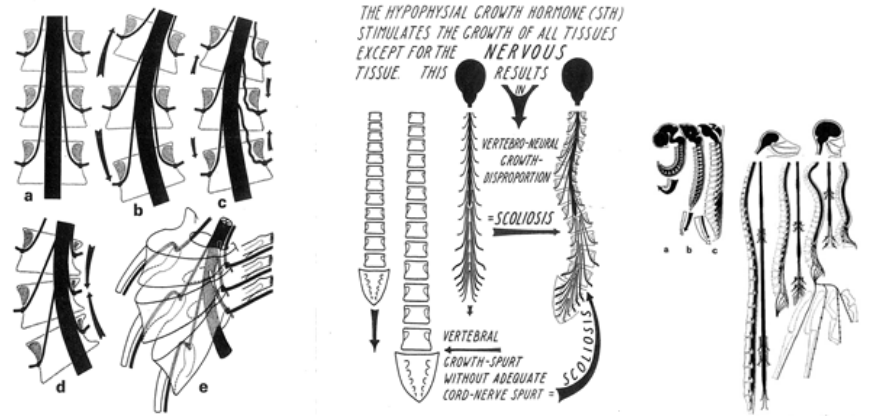

Figure 1: Some illustrative figures by Roth in which he made drawings of the concepted theory on the discongruent growth beteween the osseous and nervous tissues based on his observations as a neuroradiologist. (courtesy mrs. Rothova). 
on the musculoskeletal apparatus and/or in serious cases by intrinsic factors like lack of oxygen, infections or drugs, that impair the healthy growth of nervous tissue intracellular.

Bipedalism in men demands a huge effort to the whole bodily system: how do we keep that heavy skull in the most optimal balance and keep the whole mechanism durable without loss of too much energy. An optimal posture and curvature will be beneficial for the proper development and durable function of all other organ systems too as it is for an optimal locomotion in gait and running. It is the cultural change in lifestyle around sitting that puts us progressively in flexed positions, that hinders the normal morphogenesis as well as the physiogenesis.

Recently, the Hong Kong group lead by Cheng and Chu published supportive reproducing studies on Roth's observations with MRI and neurosignals in idiopathic scoliosis [20,21]. Such research in scoliosis should be done in children with kyphotic deformities too, to establish a same etiologic, only not asymmetrical, pathway of morphogenesis [22].

MRI imaging gives us the opportunity to visualize simultaneously what happens with the cord and roots, the vertebrae, and the muscles involved. In research MRI-imaging should become a new standard in assessing what to look for and how to measure and to interpret it. But all imaging is just a momentum in longstanding processes. For the ongoing processes in time, the fourth dimension, one has to understand Roth's concepts.

Milan Roth published his views on the causation of scoliosis stating that:

"Idiopathic scoliosis may be interpreted as an adaptive morphogenetic reaction of the vertebral column upon the growth insufficiency of the intraspinal nervous structures: The growth process of the vertebral column, though continuing undisturbed at the cellular level, is adapted at the organ level by "waves" to the growth-insufficient cord-nerve complex with the musculature as the force delivering organ. Morphological features of the scoliotic vertebra together with the typical position of the spinal cord within the spinal canal speak in favour of the suggested vertebraneural concept which offers a plausible explanation of the congenital and experimental scoliosis as well".

As life and growth in Nature are always arranged as optimisation processes, he reached the base of the biology of vertebrates. Genetics plays its role, but more in terms of delivering characteristics and qualities like resilience to all sorts of mechanical forces in all cells and not as predicting blueprint. Ailments and detoriations are end stages of multifactor processes. Form follows function. Darwin would agree on this.

\section{How does this Disproportionate Growth Work out at the Level of the Vertebrae and Musculature?}

Growth to complete structural integrity means that muscular strength, fascial and tendon length, ROM of all synovial joints and elasticity and spring like function of nonsynovial connections like intervertebral discs, symphysis etc. must be optimalised in their relationship. Disproportionate growth means that the balanced interrelationship between the involved tissues must be disturbed. In the absence of intrinsic stretch ability of nerves as in CP, you see detoriation in all tissues. If there is increased tension in the cord, while its growth is not in pace with the increased length of the skeleton, there must be increased tension in muscles too. This energy of muscles that try to stop skeletal length is capable to deform the soft connecting parts (Volkmann Hueter principle) or the immature bones (Wolff's Law) of the spine. This tension or thightness in muscles at the backside of the body (erector spinae, hamstrings, and triceps surae) is well known in Scheuermann disease, spondylolisthesis, and juvenile disc herniation but never seen as a functional part of these deformations [20]. Lambrinudi described this tension already in scoliosis too, as we did in 2008 [21,22]. Tension (overstretching) is the only trigger for the nervous cell in the extremities that gives a response by reflexes. The accurate assessment of this tension in the neuromuscular system has the promise of giving us the "early signs" of starting deformations and the precursor of the feared progression. At the other hand proper assessment of the developing posture is possible as well in standing, sitting and bend position. We know to what criteria a good or optimal curvature in the spine should meet. Semi recent literature on good postures is very scarce and not informative what the found variation in (mostly radiologic) measurements of curvature means [23-25]. The variation in curvature in early adulthood should be seen as a resultant of all growing processes and not as a given starting point or genetically desired endpoint. The cord and roots "strive" for a complete central position within the bony structures to arrange at the end of growth enough space at all sides along their course. Roth revised Holtzer's neuroprotective mechanism on this. The visible spiralization in scoliosis with its apex in a hypokyphotic region is also according to Jansen and only proven by Dickson and his followers in the thoracic spine, because they overlooked apparently the events at the thoracolumbar spine [26]. One of the main explanations can be, looking to the musculature, that in developing kyphosis the proper deepening of the vertebral groove besides the spinal processus become shallow, and with only slight, but persistent torsional forces (Jansen) the erector trunci will slip gradually out of this groove (Figures 2 and 3). The theory and experiments that dorsal shear forces are likely to be present in lordotic parts of a spine still holds as recently was shown [27-29]. But in our opinion this doesn't incorporate a pre-existent kyphosis in the thoracolumbar junction and older evidence on human spinal behavior and skeletal growth. Most authors describe the dorsal shear forces in (de facto a residual) lordosis, but are not aware that this lordosis or hypokyphosis can only occur above a kyphotic change at the thoracolumbar joint. A growing vertebrate that is hindered will seek adaptations or solutions. A tree grows towards the

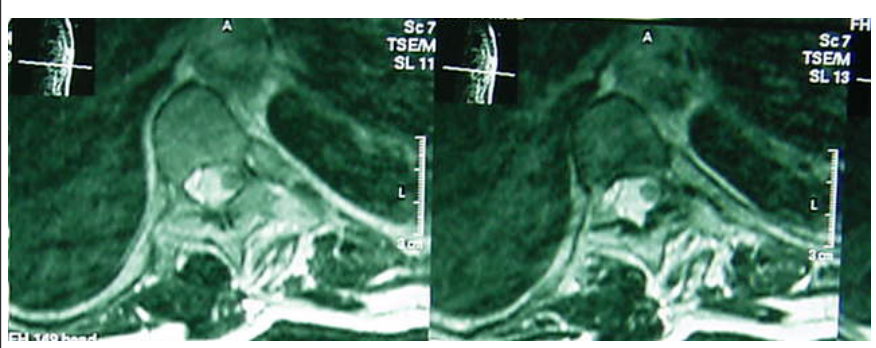

Figure 2: MRI (transverse slides) in scoliosis. According to the findings of Milan Roth with pneumomyelography studies, on MRI in the lower thoracic spine in an otherwise healthy female patient of 38 year with a never treated scoliosis, the very asymmetric position of the central cord is seen in the concavity of the curve. Also the slender caliber of the cord is not normal, as is the contact zone of the cord to the bony boundaries of the spinal canal. The picture of a bony canal that spiralizes around the precious cord (Roth), that stays by itself in the middle of the total body has in MRI its modern way of assessing concomitant features of the neural tissue in scoliosis. Of paramount importance to understand the message of Roth's work and taken consequences in TLI is the flattening of the vertebral groove at the left, concave, side (arrow) and the luxated erecting musculature (curved arrow) out of it, compared to the right side. As a cable that is dislodged from its groove in a trolley. The right muscle shows already some fatty disuse degeneration. The estimated distances from the center of the cord to the center of the muscles still show an equilateral triangle (right). 


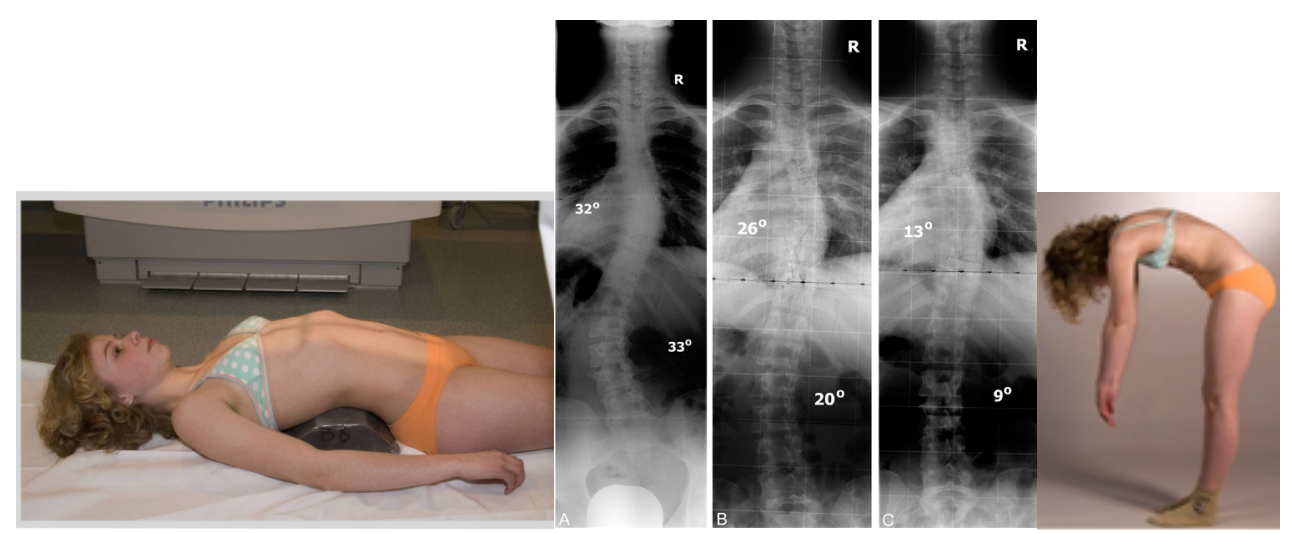

Figure 3: Figures from the experimental study in Spine to proof the potential of correction by TLI principles in double major scoliosis. In 40 children with double major scoliosis (one curve at least $25^{\circ}$ ) the Cobb angles in standing position (left X-ray) were compared with those in supine position on a lordotic fulcrum under the thoracolumbar joint (picture left and right X-ray). In all cases both curves reduced very significantly. In a subgroup a third $\mathrm{x}$ - ray was made (middle X-ray) in just supine position, because of the well-known reduction of coronal curves if gravity is ruled out (van Loon et al., [10]). The right picture is added to show the main issue of discongruent growth relations according to Roth: even this laxile girl shows thightness in the extensor muscles of the spine and legs and shows a slight kyphosis at the thoracolumbar area. (photographs by Caty Wijn).

light and will be deformed in case of a big neighbor or by too much wind which asks for adaptive solutions in further growth.

The MRI presented here under is quite informative and illustrative on how the deformative process works out. Again we see the advantages of MRI-imaging. The different aspects and actors of the process can be made visible: the position and calibre of the cord inside the canal (against the wall at the concave side of the curve), the position and deformation of the vertebra; the form of the vertebral grooves, and the left side/ right side difference of the muscles (volume, position, quality) in relation to their groove as their "cable conductor" (Figure 2).

\section{The Development of a Corrective Concept in Conservative Treatment}

On the base of the working mechanism of the formative and deformative growth we made an effort to design, develop and apply a new corrective bracing technique. In looking for supportive statements for this we found some in the work of Roth himself. First Roth is equally clear about what happens in adjusting growing forces, and the therapeutic implications this might have: "Adjustment or rectification of the deformed structure like a scoliotic spine is not accompanied by lengthening, notwithstanding the fact that the scoliotic trunk is elongated; the curved spine is adjusted, not lengthened. The adjustment involves a contraction or reduction of the convex side of the discs. Consequently in adjusting - paradoxally but irrefutably - the spinal canal is rather shortened" (End of citation).

Could we somehow use the same system (the rules of the play) to get back on track again? In our opinion Roth considered that the natural actions embodied within the Hueter-Volkmann principle could be reversed by an elastic (tensile) force. Can we use the more optimal working trajectory of the erecting muscles to realign by this the sagittal curvature of the spine? It should be noted that providing the answer to this question was not the principle object of Roth's research.

Directly supporting our observations by serendipity leading to The TLI technique, he stated that a lordotic form of the lumbar and thoracolumbar spine is of great importance for proper function of this complex and mobile region of the spine in childhood. This lordosis at the thoracolumbar spine facilitates an improved function within the lower thoracic ribcage too, where the ribs can be elevated more easily by muscular forces which also can "abduct" the ribs like in a "deep breath". The bellow-like function of the diaphragm is also optimised by this thoracolumbar lordosis by giving pretension of this muscle, what enhances the performance of a muscle.

Our conclusion was that restoring lordosis through the thoracolumbar joint appears to be the key-action to achieve the appropriate adjustments in the total, inter-connected components of the spine. The thoracolumbar joint functions in what should, or can, be seen as a three-dimensional hinge in a complex system, while it connects a pendulum(legs) with an inverted pendulum (arms), that strives to maintain a state of equilibrium in the basic or ground posture. In walking an opposite rotation occurs in the thoracic spine in comparison with the lumbar spine. Where form follows function, the core paradigm of morphogenesis, the coordinated tension of the dynamic tension cables, the spine erecting musculature, directs the positioning and form of the bony structures in the spine in order to create an intrinsically neuro-muscular well-balanced system. There is little specific systematic research to be found in the last five decades on the functioning and role of the TL-spine in non-deformed spines. The few we found give support to the specific functional aspects of this part in the pathogenesis of deformities as stated here [30].

In the found relation with the early events at the thoracolumbar joint (kyphosis in morphology, contracture in kinematics) and the possibility to come to etiology related therapeutic measurements the concept of Thoracolumbar Lordotic Intervention (TLI) was developed. In 2008 we presented a radiological study in Spine, demonstrating that forceful restoration of lordosis with a fulcrum under the thoracolumbar spine in supine children with double major scoliosis can correct instantaneous both curves significantly (Figure 3) [31]. Also, for the first time, a consequent kyphotic curve instead of the expected lordosis (in scoliosis) was found and described between the two coronal curves at the thoracolumbar spine in standing lateral radiographs. This finding is reproduced very recently by the study group of Shanghai [32].

\section{Can this Instant Reduction and De-Spiralization be Transformed in Structural Corrective Growth with Help of a Brace?}

But how promising applying such a forced lordosis looks, it was still just an instant "switch" back to a desired or more natural position. The 
study was not a proof of structural, durable corrective growth. Because trying to preserve this $3-\mathrm{D}$ reduction into real corrective growth is a severe challenge as the prevailing method in bracing tries to bring the thoracic spine into kyphosis! Here we come up to an essential statement of Dickson that can be seen as a watershed in post-world war II developments. This is formulated in the key article by Dickson in The Lancet of 1988, from which the following citation comes:

"Treatment considerations: The kyphotic spine clearly requires extension so that the anterior aspects of the growth plates can be unloaded, and if a brace or cast which maintains spinal extension is applied then there is true physiological correction of sagittal spinal shape. Scheuermann disease, therefore, is eminently treatable conservatively. The thoracic lordosis of idiopathic scoliosis would, however, require flexion and this is precisely why the deformity is not treatable conservatively and ...... There is no clear evidence that it alters the natural history of the condition. The deformity of idiopathic scoliosis can, therefore, only be corrected surgically." (End of citation)

In the challenge this quote gave us, TLI was investigated in our brace. Because by forcing the erecting muscles in their normal track or gulley and stretch or extend the spine by that we have to face the fact that this position adds to some "instability" in the thoracic facet joints. These muscles are relatively floppy in the first period in this position; already deformed vertebrae (adjusted to the curvature prevailing loading patterns) can make this position theoretically unstable.

Wearing a brace with TLI principles that holds the structure in the same stabilized position seems the answer. Assuming of course we would be able to conquer this inherent threat of instability. Dickson did not analyze or explore the possibility of such a solution; let alone that he would find evidence for its theoretical background. They have to stick to the events visible on the X-ray that shows a "hypokyphosis" or lordosis at the apex zone of thoracic curves in scoliosis.

In wearing a TLI brace the erecting muscles are guided and stimulated during the first period to adapt to the new (but more natural ) situation In this situation in which further spinal growth will be consolidated in such a span of time that a durable despiralization will be part of the correction too. The theoretical increased instability in the apical regions will disappear if the bone- and disc modelling gets structural. Of course a remaining potential of fast spinal growth in the growth spurt will act as a powerful ally (as is proper compliance) to get as much correction as possible at the end of growth. Besides wearing a brace the musculature must be trained for these "new" positions. Because of the dynamic features of the technique the condition of the muscles will improve, but postural training as in Pilates or successors of the former German "Heilgymnastik" is advisable. Our corrective principle is based on the knowledge of these methods of exercise too.

\section{The Design of as Corrective Brace}

The TLI brace that was developed and in use now applies a complete controlled lordosis at the Th12-L1 level and gives an extra strain on the erector trunci through pads at the backside only to avoid temporary flabbiness of these muscles that will use this fulcrum for increase of its performance. The normal growth forces and the proper function of the spine erecting muscles are brought back in their anatomical tracts. The human spine is the only one that has such trails in the form of the "vertebral grooves", the concavities (incisurae vertebralis) at each side of the spinal processes in the vertebral laminae [33]. And as a second working principle The TLI brace prevents flexion, the rapidly increased and most prevailing "posture" in modern life of children. The German "Sitzbuckel" has its modern counterpart in "the Gameboy back". The more laxile children are in their soft parts of the system, and girls are genetically, the more chance the ever present rotational forces in the body have to seek pathways of lesser resistance. The constant reduction towards the optimal balanced and symmetric posture will last as was shown in our study in Scoliosis Journal in 2012 [34]. But success will depend on the willingness of any individual with a deformity to wear such a brace for a prolonged time.

\section{Characteristics of the TLI Brace}

In TLI-bracing we do not put corrective force on the bony structures, but more logically, stimulate the tension cables of a flexible structure, as the posterior balancing muscles can be called, to do the job they are meant for. Rearrangement of tensile forces is required because of a complete chance that occurred in the postural balance in scoliosis or hyperkyphosis. Instead of a slight or clear flexed position of the trunk as in most TLSO's, we restore antero-posterior curvatures in that trunk, and thus, this rearrangement of masses and muscle forces, important to control the balance will end in a corrected three-dimensional posture. By achieving a more balanced posture the presence or need of "tight hamstrings" disappears (Tensegrity). We gently force the child's growing body to auto correct the deformity while we focus on intervening only in the sagittal plane. Shoulders and buttocks regain their relative posterior position, while the thoracolumbar area is passively and actively placed at a more anterior position.

Not part of this article, but important to mention is that we under scribe the importance and use of early posture devoted Physiotherapy, and continue this while wearing braces to optimalise strength and active postural balance during all sorts of activities. As an orthosis is a prescribed device, which must be understood by physicians, paramedics and patients, some description is given.

\section{Characteristics of the Device}

Solid brace of PE with preformed lordosis with the apex at the TL area. Since 2014 the braces are moulded in a customised way after 3D scanning and by CadCam methods ( ProReva Apeldoorn). The first amount of lordosis at the TL joint is prescribed by the orthopaedic surgeon on base of a special assessment of the correctabilty in a sitting bending test. There is complete left/right symmetry offered to all spines (Figure 4). TLI braces are open at the back; so there is no direct contact or pressure on the spinous processes or the ribs but only on the midportion of the extending musculature. The opening at the backside provides that all counter wise movements initiated in the TL area are allowed as close as possible to movements of the joints in the

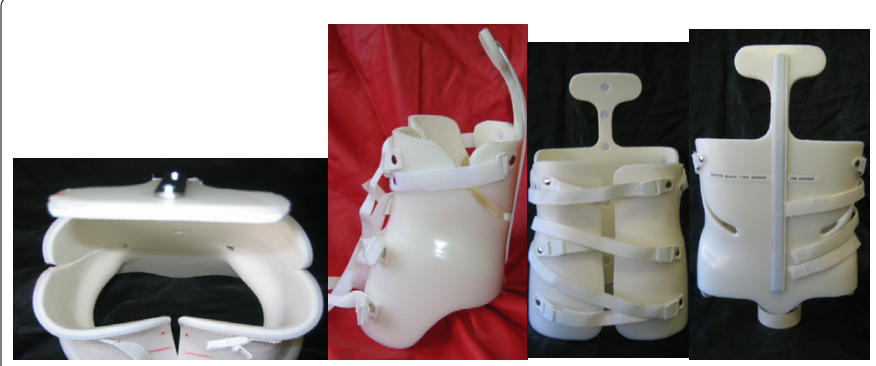

Figure 4: Several views of a TLI brace Left: A TLI brace seen from above with its backward bended sternal support. All the mobility of the thoracolumbar and thoracic spine is possible, with flexion as the only exception. The place where pressure is applied is on the paraspinal musculature at the thoracolumbar joint only. The corrective forces are symmetric and the same for all deformities (white arrows). Right: Side, front and rear view. 


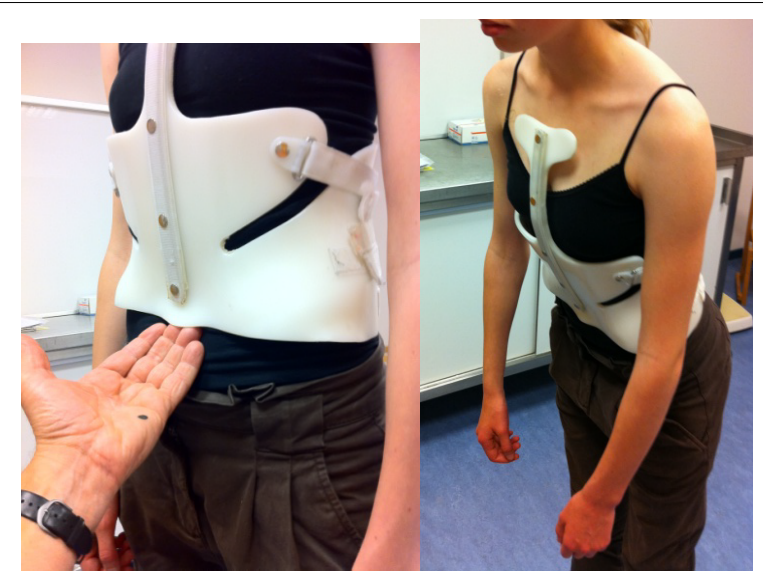

Figure 5: A girl with scoliosis in her last and smallest form of TLI brace bending a little forward to show the extension that persist in the spine that leaves the sternum free.: By automatic further extension of the torso while wearing a TLI brace, at all controls the correction in lordosis can be augmented, whilst the upper and lower rims can be shortened with a positive effect on compliance.

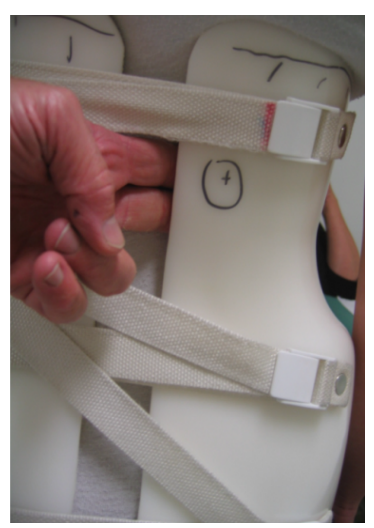

Figure 6: The extra space after the first two months of wearing. The space willed is filled up by padding. The upper rims will be shortened and bended backwards. Much of the lower rims will be taken off too.

lumbopelvic area during walking.

Rounded pelottes at the specific spots in the back in the TL area give extra strain on the erector trunci and can be made thicker at controls. A slender and bendable sternal part at the front functions as an anvil for the lordotic force (as in three point correction) and is important to prevent flexion of the spine. Grip on the iliac wings provide gross stability in the lower part and controls the pelvic torsion present in scoliosis.

\section{Advantages for Patients}

The TLI brace gives a certain freedom of movement above Th12 in all directions, except flexion (Figure 5). And is by that more dynamic than any other TLSO.

The orthosis is relatively small and its form resembles a good posture; so it less visible in wearing under clothes, what is important for good compliance. Also the instant feeling of creating "a good posture" is an important aid to compliance.

Children see and feel instant results of their body posture in relation to the brace. The autonomous despiralizing growth can be depicted by the fact that their body gets loose at points from the wall of the brace (it turns away from it) even in the first weeks. At every visit the sternum can be adjusted by bending backward, and extra padding can be added to increase the lordotic force (Figure 6).

As a result of corrective growth the brace can be made smaller at every next visit. Strips of the plastic rims at the upper part and over the buttocks can be removed to give space to pelvis and shoulders to regain their optimal and more functional position.

\section{Applicability of the Brace}

The TLI brace have been applied in more than 200 patients with scoliosis and hyperkyphosis up to now. Scoliotic curves varied from $14^{\circ}-76^{\circ}$ in the right thoracic and from $30^{\circ}-74^{\circ}$ in the lumbar left curves. Its effectiveness is fastest in children with a good remaining growth potential. We have not yet defined an optimum for age and curve pattern in relation to this effectiveness, but the adagio: as early as possible, is not disputed. Also in spondylolisthesis this brace technique offers a promise, while it will diminish the compressive forces on the posterior parts of the lumbosacral spine. The patient group that is reported in Scoliosis Journal has been treated as a consecutive group.

\section{Conclusion}

In understanding of all available and renewed knowledge on growth and how nature can provide the human spine with pathological curvatures, the TLI concepts on assessing and correcting the progressing scoliosis and kyphosis in otherwise healthy children and adolescents can be an effective tool in preventive and therapeutically strategies. The working mechanism and its relation to causative factors around the physiology of the human balance are explained.

\section{References}

1. Negrini S, Minozzi S, Bettany-Saltikov J, Zaina F, Chockalingam N, et al. (2010) Braces for idiopathic scoliosis in adolescents. Spine (Phila Pa 1976) 35: 1285 1293.

2. Rigo M, Negrini S, Weiss HR, Grivas TB, Maruyama T, et al. (2006) 'SOSORT consensus paper on brace action: TLSO biomechanics of correction (investigating the rationale for force vector selection)'. Scoliosis 1: 11.

3. Weiss HR, Goodall D (2008) Rate of complications in scoliosis surgery - a systematic review of the Pub Med literature. Scoliosis 3: 9.

4. Hohmann G, Hackenbroch M, Lindemann K (1956) Handbuch der Orthopaedie Stuttgart Georg Thieme Verlag

5. Jansen Murk: The Physiologic Scoliosis and its Cause (in Dutch) 191, Leiden E.J.Brill, publisher.

6. Mohaideen A, Nagarkatti D, Banta JV, Foley CL (2000) Not all rods are Harrington - an overview of spinal instrumentation in scoliosis treatment Pediatr Radiol 30: 110-118.

7. Wang WJ, Yeung HY, Chu WC, Tang NL, Lee KM, et al. (2011) Top theories for the etiopathogenesis of adolescent idiopathic scoliosis. J Pediatr Orthop 31: S14-27.

8. COBB JR (1958) Scoliosis; quo vadis. J Bone Joint Surg Am 40-40A: 507-10.

9. Porter RW (2001) The pathogenesis of idiopathic scoliosis: uncoupled neuroosseous growth? Eur Spine J 10: 473-481.

10. van Loon PJ, van Rhijn LW (2008) The central cord-nervous roots complex and the formation and deformation of the spine; the scientific work on systematic body growth by Milan Roth of Brno (1926-2006). Stud Health Technol Inform 140: 170-186.

11. Roth M (1985) Neurovertebral and Osteoneural Growth Relations. first ed Brno, Czech Republique: ed. J.E.Purkyne University Brno, Medical Faculty

12. Roth M (1968) Idiopathic scoliosis caused by a short spinal cord. Acta Radiol Diagn (Stockh) 7: 257-271.

13. Roth M (1971) The relative osteo-neural growth Part 1: Gegenbaurs Morph Jahrb. , Leipzig 117 (1971), S. 232-255; Part II: Gegenbaurs Morph. Jahrb. 
Citation: Loon PV, Erve RV (2015) The Development of TLI (Thoracolumbar Lordotic Intervention) as an Effective Bracing Concept for the Postural Spinal Problems - A Review. J Spine 4: 226.doi:10.4172/21657939.1000226

Page 7 of 7

Leipzig 117 (1972) , S. 312-334; Part III: Gegenbaurs Morph. Jahrb, Leipzig 117 (1972), S. 421-440

14. Roth M (1981) Idiopathic scoliosis and Scheuermann "disease": Essentially identical manifestation of neuron-vertebral growth disproportion. Radiol Diagn 22: $380-391$

15. Roth M (1969) Models of vertebro-neural relations. Acta Radiol Diagn (Stockh) 9: $746-753$.

16. Chu WC (2008) Morphological and functional electrophysiological evidence of relative spinal cord tethering in adolescent idiopathic scoliosis. Spine 33: 673680

17. Chu WC (2007) A detailed morphologic and functional magnetic resonance imaging study of the craniocervical junction in adolescent idiopathic scoliosis. Spine (Phila Pa 1976) 32: 1667-1674.

18. Roth M (1986) Cranio-cervical growth collision: another explanation of the Arnold-Chiari malformation and of basilar impression. Neuroradiology 28: 187194

19. Yamada S, Knerium DS, Mandybur GM, Schultz RL, Yamada BS (2004) Pathophysiology of tethered cord syndrome and other complex factors. Neurol Res 26: 722-726.

20. Hosman AJ, de Kleuver M, Anderson PG, van Limbeek J, Langeloo DD, et al. (2003) Scheuermann kyphosis: the importance of tight hamstrings in the surgical correction. Spine (Phila Pa 1976) 28: 2252-2259.

21. Lambrinudi C (1927) A Method of Treating Lumbar Scoliosis. Proc R Soc Med 20: $1698-1700$

22. van Loon PJ (2008) Clinical detectable tension in the growing body: new and revisited signs in clinical examination in children with postural problems and spinal deformities. Restoration of lordosis on the thoracolumbar junction can correct sagittal and coronal plane deformity; a new (revisited) linked approach on the treatment and etiology of adolescent spinal deformities. Stud Health Technol Inform 140: 52-58.
23. Fon GT, Pitt MJ, Thies AC Jr (1980) Thoracic kyphosis: range in normal subjects. AJR Am J Roentgenol 134: 979-983.

24. Bradford DS (1977) Kyphosis. Clin Orthop Relat Res : 2-4.

25. Giglio CA, Volpon JB (2007) Development and evaluation of thoracic kyphosis and lumbar lordosis during growth. J Child Orthop 1: 187-193.

26. Dickson RA (1988) The aetiology of spinal deformities. Lancet 1: 1151-1155.

27. Janssen MM, Kouwenhoven JW, Castelein RM (2010) The role of posteriorly directed shear loads acting on a pre-rotated growing spine: a hypothesis on the pathogenesis of idiopathic scoliosis. Stud Health Technol Inform 158: 112-117.

28. Kouwenhoven JW, Castelein RM (2008) The pathogenesis of adolescent idiopathic scoliosis: review of the literature. Spine (Phila Pa 1976) 33: 28982908.

29. Kouwenhoven JW, Smit TH, van der Veen AJ, Kingma I, van Dieën JH, et al (2007) Effects of dorsal versus ventral shear loads on the rotational stability of the thoracic spine: a biomechanical porcine and human cadaveric study. Spine (Phila Pa 1976) 32: 2545-2550

30. Gregersen GG, Lucas DB (1967) An in vivo study of the axial rotation of the human thoracolumbar spine. J Bone Joint Surg Am 49: 247-262.

31. Van Loon PJM (2008) Forced lordosis on the thoracolumbar junction can correct coronal plane deformity in adolescents with double major curve pattern idiopathic scoliosis. Spine 33: 797-801

32. Ni Haijian (2010) An increased kyphosis of the thoracolumbar junction is correlated to more axial vertebral rotation in thoracolumbar/lumbar adolescent idiopathic scoliosis. Spine 35: 1334-1338

33. Roth M (1969) The vertebral groove. Acta Radiol Diagn (Stockh) 9: 740-745.

34. Van Loon PJM, Roukens M, Thunnissen FB, Munneke J (201) A new brace technique for adolescent scoliosis and kyphosis based on restoration of thoracolumbar lordosis. Radiological and subjective clinical results after one year of treatment. Scoliosis, 7: 19 